\title{
Fatores associados a estresse, ansiedade e depressão em pacientes com doenças crônicas durante o período de distanciamento social
}

\author{
Factors associated with stress, anxiety and depression in patients with chronic diseases during the \\ period of social distancing
}

Factores asociados al estrés, a la ansiedad y a la depresión en pacientes con enfermedades crónicas durante el período de distanciamiento social

Recebido: 24/10/2021 | Revisado: 01/11/2021 | Aceito: 02/11/2021 | Publicado: 03/11/2021

\author{
Gustavo Fonseca de Albuquerque Souza \\ ORCID: https://orcid.org/0000-0002-1794-701X \\ Universidade Católica de Pernambuco, Brasil \\ E-mail: gugavolante@gmail.com \\ Gabriella de Almeida Figueredo Praciano \\ ORCID: https://orcid.org/0000-0002-9746-8536 \\ Universidade Católica de Pernambuco, Brasil \\ E-mail: gabriellafgpp@gmail.com \\ Laís Maciel Yamamoto Revorêdo \\ ORCID: https://orcid.org/0000-0001-7212-8853 \\ Universidade Católica de Pernambuco, Brasil \\ E-mail: laismyrevoredo@gmail.com \\ Isabelle Thays de Freitas Ramos \\ ORCID: https://orcid.org/0000-0003-1834-3311 \\ Universidade Católica de Pernambuco, Brasil \\ E-mail: isabelletfreitas@gmail.com \\ Otávio da Cunha Ferreira Neto \\ ORCID: https://orcid.org/0000-0003-0517-0212 \\ Universidade Católica de Pernambuco, Brasil \\ E-mail: otaviocferreiraneto@gmail.com \\ Juliana Soares de Oliveira Rego \\ ORCID: https://orcid.org/0000-0002-0613-8620 \\ Universidade Católica de Pernambuco, Brasil \\ E-mail: juliana_sor1@hotmail.com \\ Gabriella Viana da Costa \\ ORCID: https://orcid.org/0000-0003-2472-9819 \\ Centro Universitário Maurício de Nassau, Brasil \\ E-mail: gabriellavcosta@hotmail.com \\ Luya de Albuquerque Medeiros \\ ORCID: https://orcid.org/0000-0002-4511-8737 \\ Centro Universitário Maurício de Nassau, Brasil \\ E-mail: luyamedeiros@gmail.com \\ Alex Sandro Rolland Souza \\ ORCID: https://orcid.org/0000-0001-7039-2052 \\ Universidade Católica de Pernambuco, Brasil \\ E-mail: alexrolland@uol.com.br
}

\begin{abstract}
Resumo
Objetivo: identificar fatores associados ao estresse, ansiedade e depressão grave/extrema em doentes crônicos. Métodos: estudo de corte transversal realizado em 2020, utilizando a plataforma Google Forms contendo características sociodemográficas, hábitos de vida, questões sobre a COVID-19 e a escala de estresse, ansiedade e depressão (DASS-21). Incluíram-se 1.274 brasileiros doentes crônicos. Aplicou-se o modelo de regressão logística multivariado com nível de significância 5\%. Resultados: possuir $\leq 44$ anos, antecedentes de ansiedade e depressão, ser estudante da graduação, em isolamento social, usar medicações para dormir e reduzir/não realizar estudo, trabalho remoto e atividades de lazer possuem risco de estresse, ansiedade e depressão grave/extrema. Enquanto que ter doença cardiovascular foi protetivo para estresse (OR 0,5; IC95 \% 0,35-0,79; $p=0,002$ ) e depressão (OR 0,3; IC95\% 0,22-0,53; $p<0,0001)$ grave/extrema. Conclusão: apesar dos fatores de riscos relacionados aos doentes crônicos durante o distanciamento social, doenças cardiovasculares associou-se à proteção de transtornos mentais. Palavras-chave: Transtornos mentais; Doenças crônicas; Infecções por Coronavírus; Isolamento social.
\end{abstract}




\begin{abstract}
Objective: identify factors associated with stress, anxiety and severe/extreme depression in chronically ill patients. Methods: cross-sectional study conducted in 2020, using the Google Forms platform containing sociodemographic characteristics, lifestyle habits, questions on the COVID-19 and the stress, anxiety and depression scale (DASS-21). A total of 1,274 chronically ill Brazilians were included. Multivariate logistic regression model was applied with 5\% significance level. Results: being $\leq 44$ years old, having a history of anxiety and depression, being an undergraduate student, in social isolation, using sleeping medications, and reducing/not performing study, remote work, and leisure activities have risk of stress, anxiety, and severe/extreme depression. While having cardiovascular disease was protective for stress (OR 0.5; 95\% CI 0.35-0.79; $\mathrm{p}=0.002$ ) and depression (OR 0.3; 95\% CI 0.22-0.53; p<0.0001) severe/extreme. Conclusion: despite the risk factors related to the chronically ill during social withdrawal, cardiovascular disease was associated with protection from mental disorders.
\end{abstract}

Keywords: Mental disorders; Chronic diseases; Coronavirus infections; Social isolation.

\title{
Resumen
}

Objetivos: identificar los factores asociados al estrés, la ansiedad y depresión severa/extrema en pacientes crónicos. Métodos: estudio transversal realizado en 2020, a través de la plataforma Google Forms, con características sociodemográficas, hábitos de vida, preguntas sobre COVID-19 y escala de estrés, ansiedade, depresión (DASS-21). Se incluyeron 1.274 brasileños con enfermedades crónicas. Se aplicó un modelo de regresión logística multivariante con nivel de significación del 5\%. Resultados: tener $\leq 44$ años, antecedentes de ansiedad y depresión, estudiante de grado, aislamiento social, dormir y reducir/no realizar medicación de estudio, trabajo y actividades de ocio a distancia tienen riesgo de estrés, ansiedade, depresión severa/extrema. Tener una enfermedad cardiovascular fue protector para el estrés (OR 0,5; IC 95\% 0,35-0,79; p=0,002), la depresión (OR 0,3; IC 95\% 0,22-0,53; p<0,0001), depresión severa/extrema. Conclusión: a pesar de los factores de riesgo durante el retraimiento social, la enfermedad cardiovascular se asoció con la protección de los trastornos mentales.

Palabras clave: Trastornos mentales; Enfermedades crónica; Infecciones por Coronavirus; Aislamiento social.

\section{Introdução}

A pandemia do SARS-CoV-2 está relacionada à altas taxas de morbimortalidade, devido, principalmente, à sua elevada transmissibilidade e à indução de quadros clínicos graves. Como forma de controlar a disseminação da COVID-19, governos estabeleceram o uso obrigatório de máscara facial e medidas de distanciamento social, as quais foram caracterizadas pela redução dos contatos sociais (Aquino et al., 2020). Apesar da eficácia dessas restrições, estudos alertam sobre as consequências na saúde mental da população (Brooks et al., 2020).

A incerteza e o medo frente ao desconhecido impulsionam transformações psicossociais e emocionais, como o aumento dos níveis de estresse, ansiedade e depressão, e comportamentos adversos, como maior consumo de álcool e tabaco (Shigemura et al.,2020). Ademais, há evidências de que idosos e portadores de doenças crônicas são propensos às manifestações clínicas graves da COVID-19, necessitando de hospitalização e cuidados intensivos, e, consequentemente, possuem pior prognóstico e maior taxa de mortalidade (Eurosurveillance, 2020). Diante ao anseio de ser infectado e de desenvolver quadros mais graves, espera-se maior prevalência de sintomas psicopatológicos, como ansiedade e depressão, nesses pacientes (Wang et al., 2020).

As doenças crônicas geralmente progridem lentamente e estão associadas aos transtornos mentais, embora os sintomas psicológicos permaneçam, em sua maioria, subtratados (DeJean et al., 2013). A sintomatologia psiquiátrica advém, principalmente, do diagnóstico da doença, visto que vêm à tona sentimento de culpa, incerteza sobre o futuro, perda do senso de si e a necessidade de se isolar, seja por limitações da doença ou por sentimento de inferioridade (Willgoss et al., 2011).

A relação entre os distúrbios mentais e doenças crônicas são descritas como cíclicas. Um estudo na Filadélfia observou que a depressão e a insuficiência cardíaca estavam interligadas, sugerindo que seria necessário associar o tratamento da depressão ao cardíaco (Bogner et al., 2008). No mesmo sentido, a conectividade entre reclusão social, ansiedade e depressão estão sujeitas a um ciclo, uma vez que a sintomatologia ansiosa e/ou depressiva interfere negativamente na interação social, o que por sua vez, aumenta o sofrimento psíquico (DeJean et al., 2013). 
Visto a ligação causal entre histórico de doenças e psicopatologias (DeJean et al., 2013), bem como a situação de vulnerabilidade mental desse grupo durante o período de distanciamento social e a preocupação com o risco de infecção pelo SARS-CoV-2, o presente estudo buscou identificar os fatores associados ao estresse, ansiedade e depressão grave/extrema em doentes crônicos.

\section{Métodos}

Realizou-se um estudo de corte transversal, prospectivo (Souza et al., 2021) entre abril e maio de 2020. Foi utilizada a Plataforma Google Forms para criação do questionário online, o qual foi divulgado pelas redes sociais (Whatsapp, Facebook, Instagram e Twitter) e correio eletrônico. A divulgação ocorreu de forma sequenciada, de modo que, os integrantes da pesquisa também participaram da divulgação nas redes sociais. Incluíram-se 1274 brasileiros e portadores de doenças crônicas prévias e excluíram-se os < 18 anos ou que preenchessem o questionário de forma incompleta ou incorreta.

A amostra foi calculada no programa staltcalc do epi-info versão 3.5 .1 (centers for disease control and prevention - cdc, estados unidos da américa - eua, atlanta, dc) prevendo uma prevalência de depressão de 5\% (oms, 2017). Considerandose, ainda, um poder de $80 \%$ e um nível de significância de $95 \%$ seriam necessárias 810 pacientes, a qual foi aumentada para 1.300, prevendo-se eventuais perdas.

As variáveis estudadas foram as características biológicas, sociodemográficas, clínicas e hábitos de vida dos participantes, como variáveis independentes, além da aplicação da escala de estresse, ansiedade e depressão (DASS-21) (Vignola \& Tucci, 2014), como variável dependente. Dentre essas, temos: idade; sexo ao nascimento; religião; estado civil; região de residência no Brasil; raça/cor; número de filhos; número de cômodos em sua residência; escolaridade; área de ocupação; renda mensal; estar em distanciamento social, isolamento social ou quarentena; consumo de cigarro e bebidas alcoólicas; uso de medicamentos para dormir; prática de lazer ou exercício físico; uso da internet para conversar com amigos (redes sociais e jogos interativos); realização de trabalho ou estudo remoto; antecedentes de ansiedade/depressão; sintomas da COVID-19; doenças cardiovasculares; doenças pulmonares; diabetes; realização de teste para a COVID-19; e história de contato com alguém suspeito ou confirmado para a COVID-19.

A escala utilizada, DASS-21, é do tipo Likert com quatro pontuações, que variam entre zero (não se aplicou nada em mim), um (aplicou-se a mim algumas vezes), dois (aplicou-se a mim muitas vezes) e três (aplicou-se muito a mim a maior parte das vezes). As pontuações para depressão, ansiedade e estresse são definidas pela soma dos escores, sendo sete para cada um dos transtornos, dos 21 itens totalizados. Para interpretação, foi adotado os escores de depressão com pontuações de zero a nove indicando normalidade, 10 a 13 depressão leve, 14 a 20 moderada, 21 a 27 grave e a partir de 28 extrema. Para a análise da ansiedade a pontuação de zero a sete indica normalidade, oito a nove ansiedade leve, 10 a 14 moderada, 15 a 19 grave e a partir de 28 extrema. Por fim, para a análise do estresse, a pontuação de zero a 14 indicou normalidade, 15 a 18 estresse leve, 19 a 25 moderado, 26 a 33 grave e a partir de 34 extremo (Vignola \& Tucci, 2014).

O estudo ocorreu após a aprovação Comité de Ética em Pesquisa (CEP) em Seres Humanos da Universidade Católica de Pernambuco (UNICAP) com número de parecer de 3.988.875 de 24 de abril de 2020 e CAAE: 30623020.1.0000.5206. Todos os participantes concordaram e assinaram eletronicamente o Termo de Consentimento Livre e Esclarecido (TCLE).

A análise estatística foi realizada pelo software Epi-info 7.2.4.0 (CDC, Atlanta, DC). A variável idade foi expressa em média e desvio padrão e as categóricas em número absolutos e distribuição de frequência. Realizou-se análise para testar a associação das variáveis dependentes e independentes na população estudada, pelo teste de chi-quadrado, sendo calculada a força dessa associação pela razão de prevalência e seu intervalo de confiança a 95\% (IC95\%).

Em seguida foi feita a análise de regressão logística multivariada, com o modelo inicial sendo composto pelas variáveis explanatórias que apresentaram valor $p<0,20$ na análise univariada, calculando-se o Odds Ratio (OR) inicial e seu 
IC95\%. Para o modelo final, permaneceram as variáveis com nível de significância menor que $0,05(p<0,05)$, sendo calculado o OR ajustado e seu IC95\%.

\section{Resultados}

Foram obtidas 1.300 respostas no questionário, dentre as quais 1274 (98,0\%) foram incluídas no estudo. Excluíram-se $20(1,5 \%)$ por preenchimento incompleto e seis $(0,5 \%)$ por possuírem menos de 18 anos.

Identificou-se uma média de idade de 35,3 \pm 14,1 anos e dentre as variáveis estudadas a maioria era do sexo feminino ( $\mathrm{n}=966 ; 75,8 \%)$. Quanto as doenças crônicas encontraram-se que, 450 (35,3\%) possuíam doenças pulmonares, 414 (32,5\%) cardiovasculares, $148(11,6 \%)$ diabetes mellitus, $86(6,7 \%)$ renais, $48(3,8 \%)$ hepáticas e $430(33,8 \%)$ outras (Tabela 1).

Tabela 1. Perfil dos participantes portadores de doenças crônicas, Recife, Pernambuco, Brasil, 2021.

\begin{tabular}{|c|c|c|}
\hline \multicolumn{3}{|l|}{ Variáveis } \\
\hline Idade, anos (média $\pm \mathrm{DP}$ ) & 35,3 & 14,1 \\
\hline \multicolumn{3}{|l|}{$\operatorname{Sexo}(\mathrm{n} ; \%)$} \\
\hline Feminino & 966 & 75,8 \\
\hline Masculino & 308 & 24,2 \\
\hline \multicolumn{3}{|l|}{ Raça (autodeclarada) (n;\%) } \\
\hline Branca & 765 & 60,0 \\
\hline Parda & 400 & 31,4 \\
\hline Negra & 85 & 6,7 \\
\hline Indígena & 3 & 0,3 \\
\hline Outras & 21 & 1,6 \\
\hline \multicolumn{3}{|l|}{ Religião (n;\%) } \\
\hline Católica & 565 & 44,3 \\
\hline Evangélica & 185 & 14,5 \\
\hline Espírita & 128 & 10,1 \\
\hline Outras & 81 & 6,4 \\
\hline Sem religião & 315 & 24,7 \\
\hline \multicolumn{3}{|l|}{ Escolaridade (n;\%) } \\
\hline Ensino fundamental incompleto $\left(1^{\circ}-8^{\circ}\right.$ ano $)$ & 5 & 0,4 \\
\hline Ensino fundamental completo (conclui o $9^{\circ}$ ano) & 5 & 0,4 \\
\hline Ensino médio incompleto $\left(1^{\circ}-2^{\circ}\right.$ anos $)$ & 11 & 0,9 \\
\hline Ensino médio completo (conclui $3^{\circ}$ ano) & 113 & 8,9 \\
\hline Ensino superior incompleto & 443 & 34,7 \\
\hline Ensino superior completo & 252 & 19,8 \\
\hline Pós-graduação & 445 & 34,9 \\
\hline \multicolumn{3}{|l|}{ Região do Brasil (n;\%) } \\
\hline Nordeste & 957 & 75,1 \\
\hline Sudeste & 169 & 13,3 \\
\hline Sul & 69 & 5,4 \\
\hline Centro-Oeste & 37 & 2,9 \\
\hline
\end{tabular}




\begin{tabular}{lcc} 
Norte & 29 & 2,2 \\
Outro país & 13 & 1,1 \\
Doenças crônicas (n;\%)* & & \\
Pulmonar & 450 & 35,3 \\
Cardiovascular & 414 & 32,5 \\
Diabetes Mellitus & 148 & 11,6 \\
Renal & 86 & 6,7 \\
Hepática & 48 & 3,8 \\
Outros & 430 & 33,8 \\
\hline *Participantes podem ter uma ou mais doenças crônicas. Fonte: Autores.
\end{tabular}

Quanto a prevalência de estresse, destaca-se que 628 (49,3\%) possuíam sinais e/ou sintomas psíquicos, sendo 125 $(9,8 \%)$ de gravidade extrema. Para ansiedade 601 (47,2\%) possuíam sintomas, sendo 235 (18,4\%) de gravidade moderada. Por fim, em relação à depressão 675 (53,0\%) possuíam sintomas, sendo 181 (14,2\%) de gravidade extrema (Tabela 2).

Tabela 2. Prevalência de sinais e sintomas de estresse, ansiedade e depressão, segundo a gravidade, Recife, Pernambuco, Brasil, 2021.

\begin{tabular}{ccccccccccc}
\hline & \multicolumn{2}{c}{ Normal } & \multicolumn{2}{c}{ Leve } & \multicolumn{2}{c}{ Moderada } & \multicolumn{2}{c}{ Grave } & \multicolumn{2}{c}{ Extrema } \\
\cline { 2 - 11 } Variável & $\mathbf{n}$ & $\boldsymbol{\%}$ & $\mathbf{n}$ & $\boldsymbol{\%}$ & $\mathbf{n}$ & $\boldsymbol{\%}$ & $\mathbf{n}$ & $\boldsymbol{\%}$ & $\mathbf{n}$ & $\boldsymbol{\%}$ \\
\hline Estresse & 646 & 50,7 & 173 & 13,6 & 169 & 13,3 & 161 & 12,6 & 125 & 9,8 \\
Ansiedade & 673 & 52,8 & 93 & 7,3 & 235 & 18,4 & 75 & 5,9 & 198 & 15,5 \\
Depressão & 599 & 47,0 & 167 & 13,1 & 232 & 18,2 & 95 & 7,5 & 181 & 14,2 \\
\hline
\end{tabular}

Fonte: Autores.

Após análise multivariada, permaneceram associados ao risco de estresse grave/extremo, no modelo final, idade $\leq 44$ anos (OR3,4; IC95\%2,00-5,69; $p<0,0001$ ); sexo feminino (OR2,4; IC95\%1,57-3,59; $p<0,001$ ); ser da graduação (OR1,6; IC95\%1,11-2,33; $p=0,01$ ); ausência de filhos (OR1,9; IC95\%1,33-2,86; $p=0,0006$ ); possuir antecedentes de ansiedade e depressão (OR2,4; IC95\%1,69-3,36; $p<0,001)$; iniciar, manter ou aumentar o uso de medicação para dormir (OR2,4; IC95\% 1,66-3,33; $p<0,0001$ ); não realizar ou reduzir atividades de estudo ou trabalho remoto (OR1,8; IC95\%1,27-2,49; $p=0,0008)$; não realizar ou reduzir a prática de exercícios físicos (OR1,5; IC95\%1,08-2,19; $p=0,01)$; não realizar ou reduzir práticas de lazer (OR4,0; IC95\%2,52-6,35; $p<0,0001)$; estar em distanciamento social (OR2,13; IC95\%1,16-3,91; $p=0,01$ ); e estar em isolamento social (OR1,6; IC95\%1,04-2,57; $p=0,03$ ). Enquanto, possuir história de doenças cardiovasculares (OR0,5; IC95\%0,35-0,79; $p=0,002$ ), de doenças pulmonares (OR0,4; IC95\%0,27-0,55; $p<0,0001)$ e diabetes (OR0,5; IC95\%0,26-0,88; $p=0,01$ ) foram fatores protetores para estresse grave/extremo (Tabela 3 ). 
Tabela 3. Variáveis associadas ao estresse grave/severo em pacientes com doenças crônicas durante o período de distanciamento social, Recife, Pernambuco, Brasil, 2021.

\begin{tabular}{llllllll}
\hline & \multicolumn{3}{c}{ Modelo inicial } & \multicolumn{3}{c}{ Modelo final } \\
\cline { 2 - 7 } Variável & OR & IC95\% & $\boldsymbol{p}$ & OR & IC95\% & $\boldsymbol{p}$ \\
\hline Antecedente de ansiedade e depressão & & & & & & \\
Sim & 2,4 & $1,63-3,34$ & $<0,0001$ & 2,4 & $1,69-3,36$ & $<0,0001$ \\
Não & 1,0 & & & 1,0 &
\end{tabular}

Diabetes

Sim

Não

Doenças cardiovasculares

$\operatorname{Sim}$

Não

Doenças Pulmonares

$\operatorname{Sim}$

Não

Sintomas COVID-19

Sim

Não

Sexo Feminino

$\operatorname{Sim}$

Não

Não possuo companheiro

$\operatorname{Sim}$

Não

Escolaridade

Graduando

Pós graduação

\section{Religião}

Não possuem

Possuem

\section{Redução da Renda mensal}

Sim

Não

Redução trabalho ou estudo remoto

Sim

Não

Uso de cigarro

Iniciou/aumentou/manteve

$$
\begin{array}{llllll}
0,5 & 0,27-0,92 & 0,02 & 0,5 & 0,26-0,88 & 0,01 \\
1,0 & & & 1,0 & &
\end{array}
$$

$$
\begin{array}{llllll}
0,5 & 0,35-0,80 & 0,002 & 0,5 & 0,35-0,79 & 0,002 \\
1,0 & & & 1,0 &
\end{array}
$$

$$
\begin{array}{crrrrr}
0,4 & 0,27-0,55 & <0,0001 & 0,4 & 0,27-0,55 & <0,0001 \\
1,0 & & 1,0 &
\end{array}
$$

$$
\begin{array}{lll}
1,1 & 0,65-1,77 & 0,78 \\
1,0 &
\end{array}
$$

$\begin{array}{llllll}2,3 & 1,51-3,50 & 0,0001 & 2,4 & 1,57-3,59 & <0,0001 \\ 1,0 & & & 1,0 & \end{array}$

$1,1 \quad 0,75-1,69 \quad 0,56$

1,0

$\begin{array}{llllll}1,6 & 1,08-2,32 & 0,01 & 1,6 & 1,11-2,33 & 0,01 \\ 1,0 & & & 1,0 & \end{array}$

$$
\begin{array}{lll}
1,1 & 0,78-1,63 & 0,50 \\
1,0 & \\
& & \\
1,2 & 0,87-1,66 & 0,24 \\
1,0 & &
\end{array}
$$

$$
\begin{array}{llllll}
1,8 & 1,26-2,48 & 0,001 & 1,8 & 1,27-2,49 & 0,0008 \\
1,0 & & & 1,0 &
\end{array}
$$

$0,6 \quad 0,33-1,18 \quad 0,15$ 
Diminuiu/não faz uso

\section{Exercícios físicos}

Diminuiu/não pratica

Iniciou/aumentou/manteve

Ausência de Filhos

$\operatorname{Sim}$

Não

Distanciamento social

Sim

Não

Uso de medicações para dormir

Iniciou/aumentou/manteve

Diminuiu/não faz uso

\section{Práticas de lazer}

Diminuiu/não pratica

Iniciou/aumentou/manteve

\section{Uso da internet}

Diminuiu/não pratica

Iniciou/aumentou/manteve

Idade

$\leq 44$

$>44$

\section{Quarentena}

Sim

Não

Isolamento social

Sim

Não

Morar na região nordeste

Sim

Não
1,0

$\begin{array}{llllll}1,5 & 1,07-2,19 & 0,01 & 1,5 & 1,08-2,19 & 0,01 \\ 1,0 & & & 1,0 & & \\ & & & & & \\ 1,8 & 1,15-2,83 & 0,009 & 1,9 & 1,33-2,86 & 0,0006 \\ 1,0 & & & & 1,0 & \end{array}$

$2,0 \quad 1,08-3,62 \quad 0,02$

$2,1 \quad 1,16-3,91 \quad 0,01$

1,0

1,0

$2,4 \quad 1,67-3,38<0,0001$

$2,4 \quad 1,66-3,33<0,0001$

1,0

1,0

$4,0 \quad 2,54-6,54<0,0001$

$4,0 \quad 2,52-6,35<0,0001$

1,0

1,0

$0,9 \quad 0,34-2,23 \quad 0,78$

1,0

$\begin{array}{lrlrrr}3,4 & 1,97-5,68 & <0,0001 & 3,4 & 2,00-5,69 & <0,0001 \\ 1,0 & & 1,0 & & \end{array}$

$1,1 \quad 0,70-1,63 \quad 0,74$

1,0

$1,66 \quad 1,02-2,71 \quad 0,03$

$1,6 \quad 1,04-2,57 \quad 0,03$

1,0

1,0

$0,7 \quad 0,47-0,96 \quad 0,03$

1,0

Em relação ao estresse, foram excluídos da análise multivariada, por possuir $p>0,20$, as variáveis: etnia, ser da área da saúde, quantidade de cômodos na residência, uso de bebidas alcoólicas durante a pandemia, realização de teste para a COVID-19 e história de contato com alguém suspeito para a COVID-19. Fonte: Autores.

Foram consideradas como risco para a ansiedade grave/extrema, após a análise final, idade $\leq 44$ anos (OR2,9; IC95\% 1,77-4,88; $p<0,0001)$; sexo feminino (OR2,1; IC95\%1,37-3,12; p=0,0005); ser da graduação (OR1,5; IC95\%1,02-2,10; $p=0,03$ ); redução da renda mensal durante a pandemia (OR1,4; IC95\%1,00-1,87; p=0,05); ausência de filhos (OR1,9; IC95\% 1,34-2,84; $p=0,0004)$; possuir antecedentes de ansiedade e depressão (OR2,8; IC95\% 1,97-3,88; $p<0,0001)$; não realizar ou reduzir atividades de estudo ou trabalho remoto (OR1,5; IC95\%1,10-2,14; $p=0,011$ ); iniciar, manter ou aumentar o uso de 
medicação para dormir (OR2,4; IC95\%1,73-3,41; $p<0,0001)$; não realizar ou reduzir práticas de lazer (OR2,5; IC95\%1,583,87; $p=0,0001)$ e estar em isolamento social (OR2,1; IC95\%1,34-3,18; p=0,0009). Em contrapartida, possuir diabetes (OR0,5; IC95\% 0,26-0,89; $p=0,01)$ e morar na região nordeste do Brasil (OR0,6; IC95\%0,44-0,87; $p=0,006)$ foram fatores protetores para ansiedade grave/extrema (Tabela 4$)$.

Tabela 4. Variáveis associadas à ansiedade grave/severa em pacientes com doenças crônicas durante o período de distanciamento social, Recife, Pernambuco, Brasil, 2021.

\begin{tabular}{llllllll}
\hline & & \multicolumn{3}{c}{ Modelo inicial } & \multicolumn{3}{c}{ Modelo final } \\
\cline { 2 - 7 } Variável & OR & IC95\% & $\boldsymbol{p}$ & OR & IC95\% & $\boldsymbol{p}$ \\
\hline Antecedente de ansiedade e depressão & & & & & & \\
Sim & 2,5 & $1,77-3,56$ & $<0,0001$ & 2,8 & $1,97-3,88$ & $<0,0001$ \\
Não & 1,0 & & & 1,0 & &
\end{tabular}

Diabetes

$\operatorname{Sim}$

$0,5 \quad 0,28-0,97 \quad 0,04 \quad 0,5 \quad 0,26-0,89 \quad 0,01$

Não

1,0

1,0

Doenças cardiovasculares

Sim

$0,7 \quad 0,49-1,09 \quad 0,13$

Não

1,0

Sintomas COVID-19

Sim

$1,6 \quad 1,01-2,59 \quad 0,04$

Não

Área da Saúde

Sim

$0,8 \quad 0,60-1,17 \quad 0,31$

Não

1,0

Sexo Feminino

Sim
$2,1 \quad 1,38-3,22 \quad 0,0005 \quad 2,1 \quad 1,37-3,12 \quad 0,0005$
1,0
1,0

Não

Não possuo companheiro

$\operatorname{Sim}$

$0,9 \quad 0,63-1,41 \quad 0,79$

Não

1,0

Escolaridade

Graduando

$\begin{array}{llllll}1,4 & 0,93-1,98 & 0,10 & 1,5 & 1,02-2,10 & 0,03 \\ 1,0 & & & 1,0 & \end{array}$

Pós graduação

\section{Religião}

Não possuem

$1,0 \quad 0,66-1,37 \quad 0,80$

Possuem

1,0

\section{Redução da Renda mensal}

Sim

$1,3 \quad 0,98-1,85 \quad 0,06$

$1,4 \quad 1,00-1,87 \quad 0,05$

Não

1,0

1,0

Trabalho ou estudo remoto 
Research, Society and Development, v. 10, n. 14, e339101422211, 2021

(CC BY 4.0) | ISSN 2525-3409 | DOI: http://dx.doi.org/10.33448/rsd-v10i14.22211

Diminuiu/não faz uso

Iniciou/aumentou/manteve

\section{Uso de cigarro}

Iniciou/aumentou/manteve

Diminuiu/não faz uso

\section{Exercícios físicos}

Diminuiu/não pratica

Iniciou/aumentou/manteve

\section{Ausência de Filhos}

$\operatorname{Sim}$

Não

Distanciamento social

Sim

Não

Uso de medicações para dormir

Iniciou/aumentou/manteve

Diminuiu/não faz uso

\section{Práticas de lazer}

Diminuiu/não pratica

Iniciou/aumentou/manteve

\section{Uso da internet}

Diminuiu/não faz uso

Iniciou/aumentou/manteve

\section{Idade}

$\leq 44$

$>44$

\section{Quarentena}

Sim

Não

\section{Isolamento social}

Sim

Não

\section{Morar na região nordeste}

Sim

Não

$\begin{array}{llllll}1,5 & 1,03-2,05 & 0,02 & 1,5 & 1,10-2,14 & 0,011 \\ 1,0 & & & 1,0 & \end{array}$

$1,4 \quad 0,78-2,57 \quad 0,24$

1,0

$1,2 \quad 0,84-1,69 \quad 0,31$

1,0

$\begin{array}{llllll}2,0 & 1,26-3,10 & 0,0028 & 1,9 & 1,34-2,84 & 0,0004 \\ 1,0 & & & 1,0 & \end{array}$

$1,5 \quad 0,82-2,56 \quad 0,20$

1,0

$\begin{array}{cccccc}2,5 & 1,75-3,49 & <0,0001 & 2,4 & 1,73-3,41 & <0,0001 \\ 1,0 & & 1,0 & \end{array}$

$2,3 \quad 1,45-3,70 \quad 0,0004 \quad 2,5 \quad 1,58-3,87 \quad 0,0001$

$1,0 \quad 1,0$

$1,3 \quad 0,54-3,14 \quad 0,53$

1,0

$2,6 \quad 1,58-4,43 \quad 0,0002$

$2,9 \quad 1,77-4,88 \quad<0,0001$

1,0

1,0

1,0

$\begin{array}{llllll}1,8 & 1,11-2,86 & 0,01 & 2,1 & 1,34-3,18 & 0,0009 \\ 1,0 & & & 1,0 & \end{array}$

$0,6 \quad 0,45-0,92 \quad 0,01 \quad 0,6 \quad 0,44-0,87 \quad 0,006$

$1,0 \quad 1,0$

Em relação à ansiedade grave/extrema foram excluídas da análise multivariada etnia, quantidade de cômodos na residência, uso de bebidas alcoólicas durante a pandemia, possuir doenças pulmonares prévias, realização de teste para a COVID-19 e história de contato com alguém suspeita para a COVID-19. Fonte: Autores.

Associaram-se como risco para a depressão grave/extrema as variáveis: idade $\leq 44$ anos (OR3,9; IC95\%2,16-6,94; $p<0,0001)$; não possuir companheiro(a) (OR1,9; IC95\%1,21-2,89; $p=0,004)$; ser da graduação (OR1,5; IC95\%1,01-2,23; 
$p=0,04)$; redução da renda mensal durante a pandemia (OR1,5; IC95\%1,10-2,15; $p=0,01$ ); antecedentes de ansiedade e depressão (OR2,4; IC95\%1,71-3,50; p<0,0001); não realizar ou reduzir atividades de estudo ou trabalho remoto (OR2,0; IC95\% 1,42-2,86; $p=0,0001)$; não realizar ou reduzir a prática de exercícios físicos (OR1,9; IC95\% 1,21-3,11; $p=0,005)$; iniciar, manter ou aumentar o uso de medicação para dormir (OR1,9; IC95\%1,35-2,78; $p=0,0003)$; não realizar ou reduzir práticas de lazer (OR3,1; IC95\%1,94-5,01; $p<0,0001)$ e estar em isolamento social (OR2,0; IC95\%1,26-3,22; p=0,003). Já possuir doenças cardiovasculares prévias (OR0,3; IC95\%0,22-0,53; p<0,0001) e doenças pulmonares (OR0,3; IC95\%0,18-0,38; $p<0,0001)$; e morar na região nordeste (OR0,3; IC95\%0,23-0,47; p<0,0001) foram associados à proteção para depressão grave/extrema (Tabela 5).

Tabela 5. Variáveis associadas à depressão grave/severa em pacientes com doenças crônicas durante o período de distanciamento social, Recife, Pernambuco, Brasil, 2021.

\begin{tabular}{|c|c|c|c|c|c|c|}
\hline \multirow[b]{2}{*}{ Variável } & \multicolumn{3}{|c|}{ Modelo inicial } & \multicolumn{3}{|c|}{ Modelo final } \\
\hline & $\mathbf{O R}$ & IC95\% & $p$ & OR & IC95\% & $p$ \\
\hline \multicolumn{7}{|c|}{ Antecedente de ansiedade e depressão } \\
\hline Sim & 2,2 & $1,54-3,23$ & $<0,0001$ & 2,4 & $1.71-3,50$ & $<0,0001$ \\
\hline Não & 1,0 & & & 1,0 & & \\
\hline \multicolumn{7}{|l|}{ Diabetes } \\
\hline Sim & 0,7 & $0,41-1,35$ & 0,33 & & & \\
\hline Não & 1,0 & & & & & \\
\hline \multicolumn{7}{|c|}{ Doenças cardiovasculares } \\
\hline Sim & 0,4 & $0,23-0,58$ & $<0,0001$ & 0,3 & $0,22-0,53$ & $<0,0001$ \\
\hline Não & 1,0 & & & 1,0 & & \\
\hline \multicolumn{7}{|c|}{ Doenças Pulmonares } \\
\hline Sim & 0,3 & $0,18-0,38$ & $<0,0001$ & 0,3 & $0,18-0,38$ & $<0,0001$ \\
\hline Não & 1,0 & & & 1,0 & & \\
\hline \multicolumn{7}{|c|}{ Sintomas COVID-19 } \\
\hline Sim & 1,6 & $0,92-2,61$ & 0,09 & & & \\
\hline Não & 1,0 & & & & & \\
\hline \multicolumn{7}{|c|}{ História de contato com alguém suspeita para COVID-19 } \\
\hline Sim & 0,7 & $0,46-1,10$ & 0,12 & & & \\
\hline Não & 1,0 & & & & & \\
\hline \multicolumn{7}{|c|}{ Área da Saúde } \\
\hline Sim & 0,9 & $0,60-1,24$ & 0,45 & & & \\
\hline Não & 1,0 & & & & & \\
\hline \multicolumn{7}{|c|}{ Sexo Feminino } \\
\hline Sim & 1,3 & $0,84-1,92$ & 0,24 & & & \\
\hline Não & 1,0 & & & & & \\
\hline \multicolumn{7}{|c|}{ Não possuo companheiro } \\
\hline Sim & 1,8 & $1,18-2,84$ & 0,006 & 1,9 & $1,21-2,89$ & 0,004 \\
\hline Não & 1,0 & & & 1,0 & & \\
\hline
\end{tabular}




\section{Escolaridade}

Graduando

Pós graduação

\section{Religião}

Não possuem

Possuem

\section{Redução da Renda mensal}

$\operatorname{Sim}$

Não

Trabalho ou estudo remoto

Diminuiu/não realiza

Iniciou/aumentou/manteve

Uso de bebidas alcoólicas

Iniciou/aumentou/manteve

Diminuiu/não faz uso

\section{Uso de cigarro}

Iniciou/aumentou/manteve

Diminuiu/não faz uso

\section{Exercícios físicos}

Diminuiu/não pratica

Iniciou/aumentou/manteve

\section{Ausência de Filhos}

Sim

Não

Distanciamento social

Sim

Não

Uso de medicações para dormir

Iniciou/aumentou/manteve

Diminuiu/não faz uso

\section{Práticas de lazer}

Diminuiu/não pratica

Iniciou/aumentou/manteve

\section{Uso da internet}

Diminuiu/não pratica

Iniciou/aumentou/manteve

\section{Idade}

$\leq 44$

$>44$

Quarentena

$$
\begin{array}{llllll}
1,4 & 0,90-2,06 & 0,13 & 1,5 & 1,01-2,23 & 0,04 \\
1,0 & & & 1,0 & &
\end{array}
$$

$1,3 \quad 0,91-1,92 \quad 0,13$

1,0

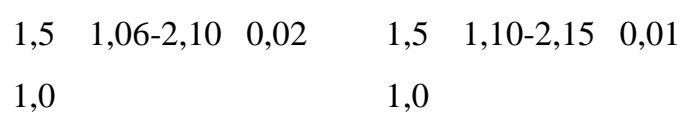

$\begin{array}{llllll}1,9 & 1,34-2,75 & 0,0003 & 2,0 & 1,42-2,86 & 0,0001\end{array}$

$1,0 \quad 1,0$

$0,8 \quad 0,55-1,11 \quad 0,17$

1,0

$0,8 \quad 0,43-1,61 \quad 0,59$

1,0

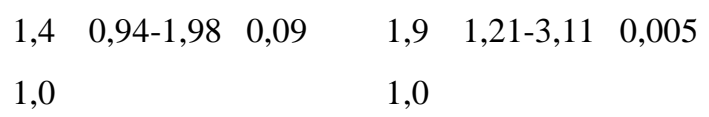

$2,0 \quad 1,23-3,27 \quad 0,004$

1,0

$1,4 \quad 0,72-2,56 \quad 0,33$

1,0

$2,0 \quad 1,41-2,96 \quad 0,0002 \quad 1,9 \quad 1,35-2,78 \quad 0,0003$

$1,0 \quad 1,0$

$3,0 \quad 1,81-4,91 \quad<0,0001 \quad 3,1 \quad 1,94-5,01 \quad<0,0001$

$1,0 \quad 1,0$

$1,1 \quad 0,42-2,86 \quad 0,82$

1,0

$3,7 \quad 2,05-6,77 \quad<0,0001 \quad 3,9 \quad 2,16-6,94 \quad<0,0001$

$1,0 \quad 1,0$ 
Sim

Não

Isolamento social

Sim

Não

Morar na região nordeste

Sim

Não
$1,2 \quad 0,79-1,91 \quad 0,34$

1,0

$1,9 \quad 1,15-3,17 \quad 0,01$

1,0

$2,0 \quad 1,26-3,22 \quad 0,003$

1,0

$0,4 \quad 0,25-0,53 \quad<0,0001 \quad 0,3 \quad 0,23-0,47 \quad<0,0001$

1,0

Em relação à depressão grave/extrema foram excluídas, na análise multivariada, as variáveis: etnia, quantidade de cômodos na residência e realização de teste para a COVID-19. Fonte: Autores.

\section{Discussão}

O presente estudo, realizado durante a pandemia, identificou alta prevalência de estresse, ansiedade e depressão, de $22,4 \%, 21,4 \%$ e $21,7 \%$, quando grave/extrema e de 49,3\%, 47,2\% e 53,0\%, quando em qualquer intensidade, respectivamente. Um estudo chinês realizado anteriormente à pandemia da COVID-19, aplicando a Escala Hospitalar de Ansiedade e Depressão (EHAD) evidenciou que, entre os portadores de doenças crônicas, 27,0\% apresentavam sintomatologia para ansiedade, 31,1\% para depressão e 20,7\% para ambos transtornos (Zhang et al., 2016). Nesse estudo chinês, as prevalências foram menores que as observadas em nosso estudo, porém maiores que a população em geral (Wang et al., 2020). Esses significativos índices podem ser explicados pelos sentimentos de tristeza, angústia e medo de ser infectado pela nova enfermidade mundial, tendo em vista a maior vulnerabilidade de quadros clínicos graves, além das restrições sociais aplicadas (Maruyama \& Ferreira, 2020).

Apesar dessa alta prevalência de transtornos mentais encontrada nos doentes crônicos, incluindo os portadores de enfermidades cardiovasculares, além do conhecimento prévio de que a população idosa é mais acometida por essas doenças e transtornos mentais (Eurosurveillance, 2020), encontramos associação de risco entre estresse, ansiedade e depressão grave/extremo com a população mais jovem, menores de 44 anos. Esse achado foi semelhante ao encontrado, durante a pandemia do SARS-CoV-2, por pesquisadores chineses (González-Sanguino et al., 2020). Isso pode ser explicado pelo menor grau de maturidade desse grupo às adversidades provocadas pela crise da COVID-19.

Outro ponto que fala a favor dessa maior maturidade encontrada na população mais idosa e portadora de doenças crônicas é que, apesar do maior risco quando comparados à população em geral, de mortalidade pela COVID-19 (Eurosurveillance, 2020) e para desenvolver estresse, ansiedade e depressão (Zhang et al., 2016), nosso estudo observou que os doentes crônicos, quando avaliado duas enfermidades separadamente, aqueles com doenças cardiovasculares, pulmonares e diabetes mellitus foram protetores para os transtornos mentais. É importante ressaltar que nosso estudo identificou os fatores de risco e protetores em portadores de doenças crônicas, incluindo as cardiovasculares, pulmonares e diabetes. Acreditamos também que essas três enfermidades prévias permitam maior suporte e apoio quando comparadas às outras doenças crônicas, uma vez que suas fisiopatologias, formas clínicas, métodos de tratamento e possibilidade de acompanhamento são mais difundidos na sociedade, com amplo tratamento no Sistema Único de Saúde do Brasil (Fittipaldi et al., 2020), o que predispõe menores índices de transtornos psíquicos. Infelizmente, poucos estudos abordam especificamente o grupo de pacientes com doenças crônicas e sua associação aos transtornos mentais (Zhang et al., 2016).

Como forma de aprofundamento sobre essa maturidade encontrada na população mais idosa e portadora de doenças crônicas, pode-se especular sobre o medo da morte. Pesquisadores estão desenvolvendo e testando escalas, como a escala de medo da COVID-19 (FCV19S), a qual avalia o medo da população frente à pandemia. Inicialmente, os grupos enquadrados 
como risco vivenciavam momentos de maior privação ao convívio social do que o resto da população, elevando, assim, a sensação de sentimentos negativos (Pereira et al., 2020). Porém, ao mesmo tempo, foi visto em estudos realizados durante a pandemia que a percepção de vivenciar uma grave ameaça seja um gatilho para a adoção de comportamentos preventivos. Em concordância com esse raciocínio, existe a teoria da motivação de proteção, de modo que os indivíduos mais suscetíveis às gravidades, como os portadores de doenças crônicas, tendem a realizar comportamentos em prol de sua saúde (Pakpour \& Griffiths, 2020).

Dentre as variáveis analisadas, o sexo feminino foi tido como preditor para estresse e ansiedade. As explicações para esse fenômeno são decorrentes de determinantes multifatoriais (Souza et al., 2020). Fisiologicamente, sabe-se que as mulheres possuem maiores níveis de estrogênios circulantes, hormônio responsável pela expressão do gene do fator de corticotropina humano (CRF), o qual é envolvido em circuitos cerebrais associados à ansiedade. Ademais, pesquisadores demonstram que as mulheres quando expostas a eventos estressores agudos, como o atual cenário de pandemia (Santos et al., 2021), e crônicos, como possuir enfermidades prévias, tornam-se mais vulneráveis a transtornos psiquiátricos (Donner \& Lowry, 2013).

A ausência de companheiro foi fator de risco para depressão grave/extrema, ao mesmo tempo em que não possuir filhos foi para estresse e ansiedade. Essas condições evidenciam a falta de interação com os familiares, em substituição às interações sociais, principalmente durante as medidas restritivas de distanciamento social, o que os expõem à solidão. A solidão e o confinamento social são fontes de estresse crônico em pacientes com doenças cardiovasculares, visto que essas circunstâncias aumentam a resistência vascular periférica (Holt-Lunstad \& Smith, 2016). Ademais, estudos demonstram haver uma significativa associação entre solidão, ansiedade e depressão, o que torna esses pacientes suscetíveis à piora clínica da enfermidade de base e ao desenvolvimento de psicopatologias (Cacioppo et al., 2015).

Manter uma vida profissional estável é um desejo da maioria dos trabalhadores, sendo esse um dos principais motivos que, a redução da renda familiar foi associada ao risco de transtornos psiquiátricos. O presente estudo foi realizado diante um cenário de distanciamento social, o que intensificou os índices de desemprego, cortes salariais e incertezas sobre a situação trabalhista, fatores que culminaram na instabilidade econômica. Além disso, outros estudos realizados com portadores de doenças crônicas constataram que as condições inerentes desse grupo, como maior cansaço e fadiga, atuam como limitantes para a realização de atividades diárias e inserção no mercado de trabalho (Hedlund et al., 2010), ou seja, maior instabilidade econômica e consequente risco para transtornos psíquicos.

Estudantes da graduação foi tido como outro fator de risco para as três psicopatologias abordadas. A entrada na vida acadêmica inclui diversas transformações no cotidiano do indivíduo, como maior sobrecarga horária, atividades extracurriculares e pressão social, desencadeando estresse, preocupação, irritabilidade e, consequentemente, quadros depressivos e ansiosos (Chatterjee et al., 2014). Os portadores de doenças crônicas vivenciam um processo contínuo e constante de mudanças, adquirindo diversos problemas. Assim, possuir um grau mais elevado de escolaridade permite que esse grupo priorize o enfrentamento dos seus problemas e, com isso, reduzam os estressores da vida diária e os índices de transtornos mentais.

Também se evidenciou risco entre o isolamento social e estresse, ansiedade e depressão grave/extrema, enquanto que o distanciamento social foi relacionado apenas ao estresse. A adesão a essas medidas restritivas mais rigorosas culmina em diminuição da interação com família e amigos, além de intensificar sentimentos de incertezas sobre o futuro, principalmente, nos grupos de risco para a COVID-19 (Brooks et al., 2020), como em pacientes diabéticos (Souza et al., 2021).

Já está consolidado que os distúrbios do sono interferem negativamente na qualidade de vida dos pacientes, estimulando sintomas psiquiátricos, morbidades e reduzindo habilidades cognitivas (Votaw et al., 2019). Evidencia-se também que o tratamento de insônia com benzodiazepínicos possui efeito limitado e pode não compensar os riscos de eventos adversos, como transtornos de humor e ansiedade, especialmente quando consumo indevido e em população de alto risco (Daré et al., 
2019). Complementando dados prévios, nosso estudo observou que os indivíduos que faziam uso de medicações para dormir estiveram vulneráveis à quadros mais graves de estresse, ansiedade e depressão.

Segundo uma meta-análise, portadores de transtornos mentais prévios são mais suscetíveis ao desenvolvimento doenças crônicas, como obesidade, doenças cardiovasculares, diabetes e, consequentemente, ao risco de possuir novas psicopatologias ou agravar às pré-existentes (Brooth et al., 2012). Confirmando esses resultados, identificou-se associação de risco entre os antecedentes de ansiedade e depressão e sintomas de estresse, ansiedade e depressão grave/extrema. Por este estudo ser de corte transversal, não foi possível determinar a relação causal entre as doenças crônicas e os transtornos mentais.

A inatividade física, condição em que os níveis de atividade física são inferiores aos necessários para uma saúde ideal e prevenção de morte prematura, é considerada precursora de doenças crônicas. Recentes trabalhos demonstram o impacto da falta de exercícios físicos sobre a saúde mental, podendo aumentar a probabilidade de desenvolvimento de estresse e depressão (Tomei et al., 2007). Aliado a isso e de acordo com nossos resultados, na presença de enfermidades é comum haver restrições das atividades diárias e de lazer, devido às limitações físicas e sentimento de culpa e de frustração, predispondo a ocorrência das três psicopatologias abordadas (Zhang et al., 2016).

O período de realização da pesquisa foi marcado por alterações nos hábitos de vida dos indivíduos, como desempregos e implementação de tecnologias para a trabalho e/ou estudo à distância (Kawohl \& Nordt, 2020). Assim como a diminuição da renda, inatividade física e práticas de lazer, a diminuição do trabalho e/ou estudo remoto foi associado ao risco de estresse, ansiedade e depressão grave/extrema. Pesquisa anterior demonstra que essa instabilidade e quebra de rotina interfere no aspecto emocional, ou seja, àqueles que não se adequaram ao novo estilo de vida apresentam maior risco para transtornos mentais (Cavalcante \& Abreu, 2020).

Estudos realizados em outros países observaram associação entre a região geográfica de residência e o risco de transtornos mentais neste período de pandemia (Cao et al., 2020), devido as regiões de maior acometimento. Seguindo essa linha, encontramos que a região nordeste do Brasil foi fator de proteção para essas psicopatologias, quando comparada as outras regiões do país. É importante destacar que dois fatores contribuíram para esses resultados. Primeiramente, por nosso estudo ter tido mais participantes da região Nordeste, seguido da região Sudeste; e por a região Sudeste ter sido a primeira a possuir altas taxas de contaminação e mortalidade, além do primeiro caso de SARS-CoV-2 no Brasil, de modo que pode ter predisposto estado de choque, angústia e distúrbios psíquicos, especialmente, em grupos de risco (Malta et al., 2019).

Os autores acreditam que, embora existam diversas variáveis temporais e espaciais da pandemia que se associam ao aumento da frequência dos sintomas psíquicos, pode-se considerar que o período de coleta de dados e o maior suporte recebido pelos pacientes, foram responsáveis pela melhor adaptação ao período estressor em que estavam submetidos, resultando em menor risco para essas psicopatologias.

Por este estudo ter sido realizado durante o período de medidas restritivas, o mesmo precisou ser realizado através de um formulário online. Tem-se o benefício com essa divulgação, alcançar uma amostra populacional maior e diversificada, porém há possibilidade de ter viés das respostas, haja vista dúvidas que os participantes possam ter tido, o que foi minimizado com a divulgação dos contatos dos pesquisadores.

Outro possível viés ocorrido foi o de seleção da amostra, a qual foi mais prevalente na região Nordeste do Brasil, local onde residiam os pesquisadores. Isso foi reduzido com as constantes chamadas e envio da pesquisa pelas redes sociais para grupos de todos os estados brasileiros.

Neste estudo não houve uma abordagem com especificidade sobre as doenças, pois pretendia-se avaliar o efeito do distanciamento social nesse período da pandemia da COVID-19, assim o tratamento realizado, gravidade e regularidade das consultas não foram abordadas. 
Por se tratar de um estudo transversal a relação de casualidade entre as doenças crônicas e os transtornos mentais não foi possível de ser determinada, sugerindo a realização de estudos observacionais de coorte ou ensaios clínicos randomizados, para avaliar a efetividade do tratamento desses transtornos mentais durante a pandemia nesse perfil de paciente.

É perceptível o crescente número de doentes crônicos e o incentivo à realização de estudos direcionados para a saúde mental dessa população. Diante desses fatos, ao avaliar a condição psíquica desses indivíduos, foi encontrado altas taxas de estresse, ansiedade e depressão grave/extrema. Observou-se ainda que possuir $\leq 44$ anos, ser estudante da graduação, possuir antecedentes de ansiedade e depressão, estar em isolamento social, fazer uso de medicações para dormir e reduzir ou não realizar estudo ou trabalho remoto e atividades de lazer, foram fatores preditivos para as três psicopatologias.

\section{Conclusão}

Assim, diante dos fatores encontrados e seguindo o conceito de saúde, que engloba o bem estar físico e mental, é de extrema necessidade o apoio a essa população, mesmo que remotamente, por equipe multiprofissional, atuando nos pilares: físico, por meio de endocrinologistas, cardiologista, clínicos e pneumologistas; mental, por psicólogos e psiquiatras; relaxamento, por educadores físicos, terapeutas ocupacionais e diversas áreas; espiritual, por lideranças religiosas; e econômico, por conselheiros financeiros. Ademais, sugere-se que novos estudos sejam realizados com um tamanho amostral maior e com uma população com maior diversidade, a fim de que resultados com maior nível de evidência sejam obtidos.

\section{Referências}

Aquino, E. M. L; Silveira, I. H.; Pescarini, J. M.; Aquino, R..; Souza-Filho, J. \& Rocha, A. S., Ferreira, A.; Victor A.; Teixeira C.; Machado D. B.; Paixão E.; Alves, F. J. O.; Pilleco, F.; Menezes, G.; Gabrielli, L.; Leite, L.; de Almeida, M. C. C.; Ortelan, N.; Fernandes, Q. H. R. F.; Ortiz, R. J. F.; Palmeira, R. N.; Pinto Junior, E. P.; Aragão, E.; de Souza, L. E. P. F.; Netto, M. B.; Teixeira, M. G.; Barreto, M. L.; Ichihara, M.Y. \& Lima, R. T. R. S. (2020). Medidas de distanciamento social no controle da pandemia de COVID-19: potenciais impactos e desafios no Brasil. Ciênc. saúde coletiva, 25: 24232446. https://doi.org/10.1590/1413-81232020256.1.10502020.

Bogner, H. R.; Dahlberg, B.; de Vries, H. F.; Cahil, 1. E. \& Barg, F. K. (2008). Older patients' views on the relationship between depression and heart disease. Fam Med, 40:652-7. https://pubmed.ncbi.nlm.nih.gov/18830841/.

Booth, F. W.; Roberts, C. K. \& Laye, M. J. (2012). Lack of exercise is a major cause of chronic diseases. Compr Physiol, 2:1143-211. https://doi.org/10.1002/cphy.c110025.

Brooks, S. K.; Webster, R. K.; Smith, L. E.; Woodland, L.; Wessely, S. \& Greenberg, N. (2020). The psychological impact of quarantine and how to reduce it: Rapid review of the evidence. Lancet, 395:912-920. https://doi.org/10.1016/S0140-6736(20)30460-8.

Cacioppo, J. T.; Cacioppo, S.; Cole, S. W.; Capitanio, J. P.; Goossens, L. \& Boomsma, D. I. (2015). Loneliness across phylogeny and a call for comparative studies and animal models. Perspect Psychol Sci, 10:202-212. https://doi.org/10.1177/1745691614564876.

Cao, W.; Fang, Z.; Hou, G.; Han, M.; Xu, X. \& Dong, J. (2020). O impacto psicológico da epidemia COVID-19 em estudantes universitários na China. Psiquiatria Res, 287: 112934. https://doi.org/10.1016/j.psychres.2020.112934.

Cavalcante, J. R. \& Abreu, A. J. L. (2020). COVID-19 no município do Rio de Janeiro: análise espacial da ocorrência dos primeiros casos e óbitos confirmados. Epidemiol. Serv. Saúde, 29: e2020204. https://doi.org/10.5123/S1679-49742020000300007.

Chatterjee, S.; Saha, I.; Mukhopadhyay, S.; Misra, R.; Chakraborty, A. \& Bhattacharya, A. (2014). Depression among nursing students in a Indian government college. Br J Nurs, 23:316-20. https://doi.org/10.12968/bjon.2014.23.6.316.

Daré, L. O.; Bruand, P. E.; Gérard, D.; Marin, B.; Lameyre, V.; Boumédiène, F. \& Preux, P. M. (2019). Co-morbidities of mental disorders and chronic physical diseases in developing and emerging countries: a meta-analysis. BMC public health, 19: 304. https://doi.org/10.1186/s12889-019-6623-6.

DeJean, D.; Giacomini, M.; Vanstone, M. \& Brundisini, F. (2013). Patient experiences of depression and anxiety with chronic disease: a systematic review and qualitative meta-synthesis. Ont Health Technol Assess Ser, 1:1-33. https://pubmed.ncbi.nlm.nih.gov/24228079/.

Donner, N. C. \& Lowry, C. A. (2013). Sex differences in anxiety and emotional behavior. Pflugers Arch, 465:601-626. https://doi.org/10.1007/s00424-013$1271-7$.

Eurosurveillance Editorial Team. (2020). Updated rapid risk assessment from ECDC on coronavirus disease 2019 (COVID-19) pandemic: increased transmission in the EU/EEA and the UK. Euro Surveill, 25:2003121. https://doi.org/10.2807/1560-7917.ES.2020.25.12.2003261.

Fittipaldi, E. O. S.; Andrade, A. D.; Santos, A. C. O.; Campos, S.; Fernandes, J. \& Catanho, M. T. J. (2020). Sintomas depressivos estão Associados a Níveis Séricos Elevados de Colesterol de Lipoproteína de Baixa Densidade em Idosos com Diabetes Mellitus Tipo 2. Arq. Bras. Cardiol, In Epub July 15. https://doi.org/10.36660/abc.20190404. 
González-Sanguino, C.; Ausín, B.; Castellanos, M. Á.; Saiz, J.; López-Gómez, A. \& Ugidos, C. (2020). Mental health consequences during the initial stage of the 2020 Coronavirus pandemic (COVID-19) in Spain. Brain Behav Immun, 87:172-176. https://doi.org/10.1016/j.bbi.2020.05.040.

Hedlund, M.; Zetterling, M.; Ronne-Engstrom, E.; Ekselius, L. \& Carlsson, M. (2010). Perceived recovery after aneurysmal subarachnoid haemorrhage in individuals with or without depression. J Clin Nurs, 19:1578-87. https://doi.org/10.1111/j.1365-2702.2009.02940.x.

Holt-Lunstad, J. \& Smith, T. B. (2016). Loneliness and social isolation as risk factors for CVD: implications for evidence-based patient care and scientific inquiry. Heart, 102:987-9. https://doi.org/10.1136/heartjnl-2015-309242.

Kawohl, W. \& Nordt, C. (2020). COVID-19, unemployment, and suicide. The lancet. Psychiatry, 7: 389-390. https://doi.org/10.1016/S2215-0366(20)301413

Malta, D. C.; Andrade, S. S. C.; Oliveira, T. P.; Moura, L.; Prado, R. R. \& Souza, M. F. M. (2019). Probability of premature death for chronic noncommunicable diseases, Brazil and Regions, projections to 2025. Rev. bras. Epidemiol, 22: e190030. https://doi.org/10.1590/1980-549720190030

Maruyama, M. E. B. \& Ferreira, H. G. (2020). Saúde mental e doenças crônicas em idosos de um grupo hiperdia. REFACS, 8: 600-611. https://doi.org/10.18554/refacs.v8i0.4696.

Pakpour, A. H. \& Griffiths, M. D. (2020). The fear of COVID-19 and its role in preventive behaviors. Journal of Concurrent Disorders, 2020; TBD: TBD. http://irep.ntu.ac.uk/id/eprint/39561.

Pereira, M. D.; Oliveira, L. C.; Costa, C. F. T.; Bezerra, C. M. O.; Pereira, M. D. \& Santos, C. K. A. (2020). The COVID-19 pandemic, social isolation, consequences on mental health and coping strategies: an integrative review. Research, Society and Development, 9: 1-35, e652974548. https://doi.org/10.33448/rsd-v9i7.4548.

Santos, A. C.; Tenorio, P. J.; Barbosa, E. A. S.; Souza, G. F. A.; Souza G. A.; Praciano, G. A. F.; Maranhão, L. B. B.; Costa, G. O. L. P. \& Souza, A. S. R. (2021). The testing for COVID-19 in symptomatic patients as a protective factor against stress, anxiety, and depression. Revista Brasileira de Saúde Materno Infantil. 21(Suppl1):133-143. https://doi.org/10.1590/1806-9304202100S100007>.

Shigemura, J.; Ursano, R. J.; Morganstein, J. C.; Kurosawa, M. \& Benedek, D. M. (2020). Public responses to the novel 2019 coronavirus (2019-nCoV) in Japan: mental health consequences and target populations. Psychiatry Clin Neurosci, 74:281-2. https://doi.org/10.1111/pcn.12988.

Souza, A. S. R; Souza, G. F. A.; Souza G. A.; Cordeiro, A. L. N.; Praciano, G. A. F.; Alves, A. C. S.; Santos, A. C.; Silva Júnior, J. R. \& Souza, M. B. R. (2021). Factors associated with stress, anxiety, and depression during social distancing in Brazil. Rev Saude Publica, 55:5. https://doi.org/10.11606/s15188787.2021055003152 .

Souza, A. S. R; Souza, G. F. A. \& Praciano, G. A. F. (2020). A saúde mental das mulheres em tempos da covid-19. Rev. Bras. Saude Mater. Infant, 20(3). https://doi.org/10.1590/1806-93042020000300001.

Souza, G. F. A.; Praciano, G. A. F.; Ferreira Neto, O. C.; Paiva, M. C.; Jesus, R. P. F. S.; Cordeiro, A. L. N.; Souza, G. A.; Silva Junior, J. R. \& Souza, A. S. R. (2021). Factors associated with psychic symptomatology in diabetics during the COVID-19 pandemic. Revista Brasileira de Saúde Materno Infantil, 21(Suppl1):177-186. https://doi.org/10.1590/1806-04202100S100009>.

Tomei, G.; Cangemi, C.; Giubilati, R. \& Fioravanti, M. (2007). Salute mentale ed alterazione delle abitudini di vita dei lavoratori giovani [Mental health and life style changes in young workers]. G Ital Med Lav Ergon, 29:903-921. https://gimle.fsm.it.

Vignola, R. C. \& Tucci, A. M. (2014). Adaptation and validation of the depression, anxiety and stress scale (DASS) to Brazilian Portuguese. J Affect Disord, 155: 104-109. https://doi.org/ 10.1016/j.jad.2013.10.031.

Votaw, V. R.; Geyer, R.; Rieselbach, M. M. \& McHugh, R. K. (2019). The epidemiology of benzodiazepine misuse: A systematic review. Drug Alcohol Depend, 200:95-114. https://doi.org/10.1016/j.drugalcdep.2019.02.033

Wang, B.; Li, R.; Lu, Z. \& Huang, Y. (2020). Does comorbidity increase the risk of patients with COVID-19: evidence from meta-analysis. Aging (Albany NY), 12:6049-6057. https://doi.org/10.18632/aging.103000

Willgoss, T.; Yohannes, A.; Goldbart, J. \& Fatoye, F. (2011). COPD and anxiety: its impact on patients' lives. Nurs Times. 107:16-9. https://pubmed.ncbi.nlm.nih.gov/21661487/.

World Health Organization. (2017). Depression and Other Common Mental Disorders: Global Health Estimates. Geneva: World Health Organization. https://apps.who.int/iris/handle/10665/254610.

Zhang, A. Z.; Wang, Q. C.; Huang, K. M.; Huang, J. G.; Zhou, C. H. \& Sun, F. Q. (2016). Prevalence of depression and anxiety in patients with chronic digestive system diseases: A multicenter epidemiological study. World journal of gastroenterology, 22: 9437-9444. https://doi.org/10.3748/wjg.v22.i42.9437. 